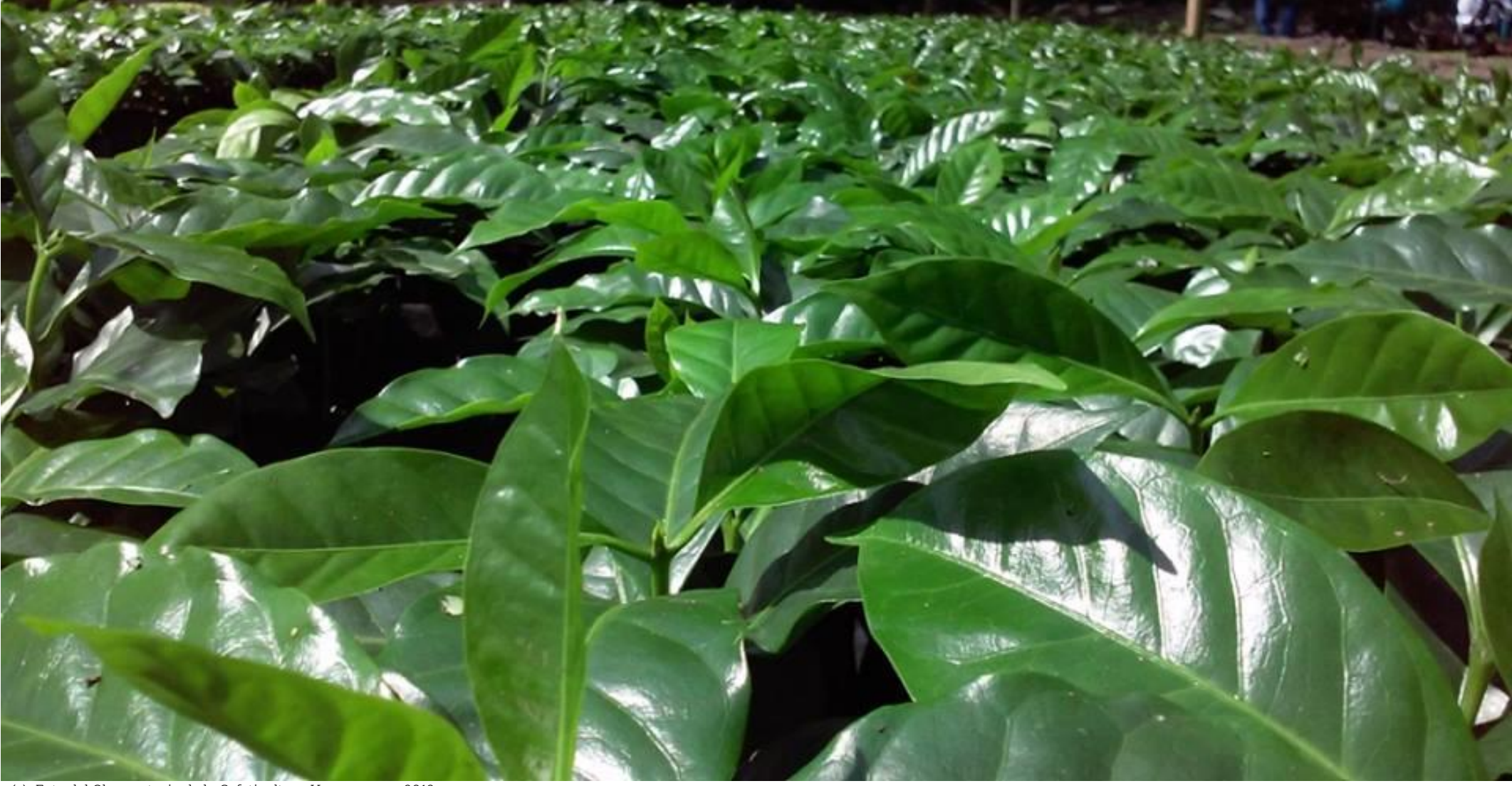

\title{
Fenómenos meteorológicos y su efecto sobre la producción de café en la Zona Central de Veracruz
}

Paulo César Parada Molina ${ }^{a}$-Mario Javier Gómez Martínez ${ }^{\mathrm{b}}$ - Gustavo Celestino Ortiz Ceballos $^{c}-$ Carlos Roberto Cerdán Cabrerad $^{d}$ Juan Cervantes Pérez ${ }^{\mathrm{e}}$

RESUMEN: La producción de café se está viendo afectada por cambios en los patrones climáticos y por la aparición de fenómenos meteorológicos que coinciden con importantes etapas fenológicas para este cultivo. El café es más sensible a variaciones del clima cuando se encuentra en las etapas de floración y el inicio del crecimiento del fruto, en este periodo cuatro fenómenos están presentes, que de acuerdo con su intensidad podría incidir de manera negativa. La etapa de maduración y cosecha es impactada por cinco fenómenos mientras que

\footnotetext{
${ }^{a}$ Facultad de Ciencias Agrícolas, región Xalapa, Universidad Veracruzana.

b Facultad de Ciencias Agrícolas, región Xalapa, Universidad Veracruzana. Facultad de Ingeniería Agronómica, Universidad del Tolima.

c Facultad de Ciencias Agrícolas, Xalapa, Universidad Veracruzana. Observatorio de la Cafeticultura Veracruzana, Universidad Veracruzana.

d Facultad de Ciencias Agrícolas, Xalapa, Universidad Veracruzana. Observatorio de la Cafeticultura Veracruzana, Universidad Veracruzana. contacto: ccerdan@uv.mx

${ }^{\text {e }}$ Centro de Ciencias de la Tierra, Universidad Veracruzana.

Recepción: 25/enero/2019 
durante la etapa de crecimiento y llenado del grano sólo dos fenómenos la afectan. El Niño Oscilación del Sur (ENSO), en su fase negativa, intensificaría los efectos negativos. Sin embargo, no debe pasar inadvertido los efectos positivos del ENSO, en fase fría, en la temporada seca (diciembre-abril). Se deben buscar y estudiar estrategias de mitigación a la variación climática producto de los fenómenos meteorológicos, donde los sistemas agroforestales pueden ser una alternativa para enfrentar esta problemática.

Palabras clave: Desarrollo reproductivo; ENSO; etapas fenológicas; eventos climáticos; regiones cafetaleras; variabilidad climática.

\begin{abstract}
Coffee production is being affected by changes in weather patterns and the appearance of meteorological phenomena that coincide with important phenological stages for coffee cultivation. The coffee is more sensitive to the variations of the climate when it is in the stages of flowering and the beginning of the growth of the product, in this period four phenomena are present, and according to its intensity it could have a negative impact. The stage of maturation and harvest is shocking by five phenomena while during the growth stage and the grain volumes only two phenomena stress. El Niño-Southern Oscillation (ENSO), in its negative phase, would intensify the negative effects. However, the positive effects of the ENSO, in the cold phase, during dry season (December-April) should not go unnoticed. Mitigation strategies for climate variation due to meteorological phenomena must be sought and studied, where agroforestry systems can be an alternative to address this problem.
\end{abstract}

Keywords: Reproductive Development; ENSO; Phenological Stages; Climatic Events; Coffee Regions; Climatic Variability.

\title{
Introducción
}

$\Psi$

n la actualidad se experimentan diversos cambios ambientales entre los que se encuentran el aumento en la frecuencia e intensidad de fenómenos meteorológicos, que se caracterizan por ocurrir en un lapso de unos cuantos días, a diferencia de los eventos climáticos que ocurren por períodos largos (Zarazúa et al., 2014). Además de la influencia de la variabilidad climática atribuida principalmente a El Niño/Oscilación del Sur (ENSO) y, de manera más general, al cambio climático. Estos tienen efectos negativos en los sistemas agrícolas y el nivel de afectación está en función del grado de exposición a dichos fenómenos, a la sensibilidad del cultivo y su capacidad de adaptarse a dichos estímulos o reducir sus 
efectos (IPCC, 2007). El café es uno de los principales productos negociados en los mercados mundiales y es de gran valor económico social y ambiental. Este cultivo se puede encontrar de manera frecuente en la zona central de Veracruz, estado que ocupa el segundo lugar con mayor producción de café en México, de acuerdo con Servicio de Información Agroalimentaria y Pesquera (SIAP). El área cafetalera se divide en siete regiones, distribuidas a lo largo de la cadena montañosa; entre ellas Tezonapa, Zongolica, Córdoba, Huatusco, Coatepec, Misantla y Atzalan. La mayor superficie está distribuida en las regiones de Coatepec, córdoba y Huatusco.

Todas con condiciones climáticas adecuadas para la producción de café de calidad, plantado principalmente bajo sombra de árboles (Díaz-Padilla et al., 2013). Sin embargo, el café por su rango climático limitado ha demostrado ser sensible a diversos fenómenos meteorológicos. La baja adaptación de los cafetos a estos acrecienta los impactos en su desarrollo y en consecuencia en la producción (Bunn et al., 2015; DaMatta y Ramalho, 2006).

Por sus características geográficas, esta zona se ve afectada por diversos fenómenos como la estacionalidad de las lluvias, característica de las regiones productoras de café, lluvias convectivas, lluvias orográficas, frentes fríos, eventos de norte, heladas, ondas de calor, entre otros. Estos ocasionan cambios en la cantidad, intensidad y frecuencia de eventos de precipitación, así como temperaturas extremas (Zarazúa et al., 2014), que podría generar condiciones adversas para el café (Jaramillo et al., 2009; Läderach et al., 2013). Además, la presencia del ENSO ocasiona distribuciones anómalas de variables como la temperatura, la humedad del aire, la nubosidad y las lluvias a escala estacional (Díaz y Markgraf, 2000).

Estos fenómenos son constantes durante todo el año; en algunos meses se pueden presentar varios fenómenos que podrían impactar de manera negativa el sector cafetalero. Los efectos en el cultivo de café dependen de la etapa de desarrollo en la que se encuentre el cultivo y se ven reflejados directamente en la producción; sin embargo, los efectos han sido poco reportados. Por ello, el objetivo principal de este trabajo fue analizar el desarrollo de las investigaciones en el efecto de los fenómenos meteorológicos sobre la producción de café en Veracruz, a partir de una revisión bibliográfica (1994 a 2018) para brindar una perspectiva actual del conocimiento en el tema.

\section{Fenómenos meteorológicos en la zona central de Veracruz}

Los fenómenos meteorológicos son cambios o alteraciones que ocurren de manera natural en el clima y que son considerados acontecimientos inusuales. En este sentido, las condiciones 


\section{Observatorios universitarios - Observatorio de la Cafeticultura Veracruzana}

meteorológicas y climáticas en la región montañosa central del estado de Veracruz están influenciadas por interacciones complejas entre los sistemas sinópticos, la topografía, la interacción planta-atmósfera y su proximidad al Golfo de México (Barradas et al., 2010).

Un ejemplo representativo de estas condiciones es la zona productora de Coatepec. Con un régimen de lluvias intermedias, donde las precipitaciones se presentan en verano como en invierno, observándose una temporada seca y una húmeda (Figura 1). Durante la temporada húmeda (mayo-octubre) se presentan lluvias convectivas que tienen su origen en el calentamiento superficial diurno y la humedad transportada por los vientos alisios del este; lluvias intensas ocasionadas por el paso de ondas del este y ciclones tropicales. Característico de esta zona es la sequía intraestival o canícula, que se presenta en los meses de junio a agosto. La temporada seca se caracteriza por un déficit hídrico (sequía preestival) muy marcado durante los meses de noviembre a abril y el paso de frentes fríos, que al interaccionar con la humedad del Golfo de México y la Sierra Madre Oriental producen vientos violentos del norte, lluvias ligeras, lloviznas y descenso de la temperatura (Báez et al., 1997; Holwerda et al., 2010; Muñoz-Villers et al., 2012).

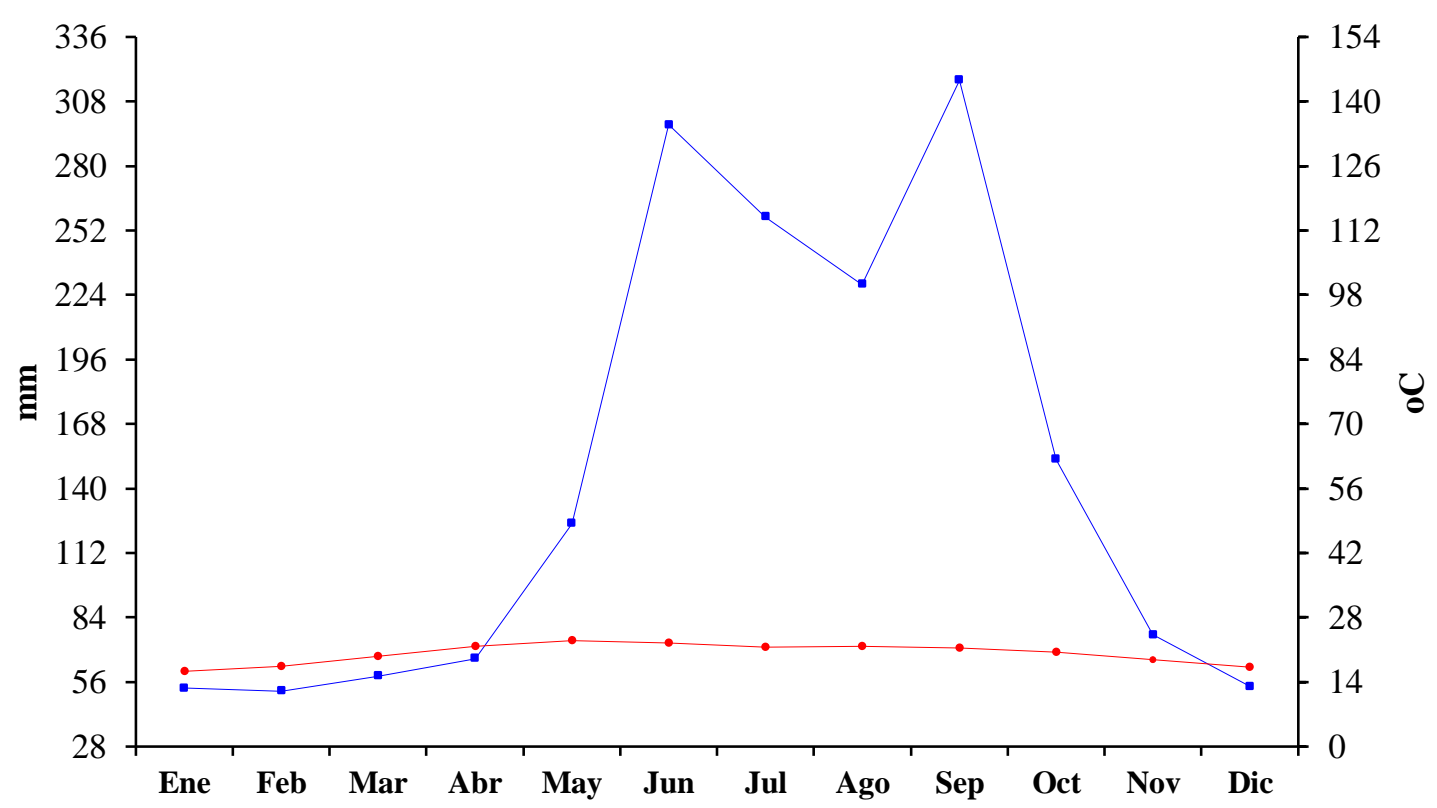

Figura 1. Temperatura promedio mensual (línea) y precipitación mensual total en la zona productora de Coatepec, zona central de Veracruz, México (1945-2013; fuente de datos: Servicio Meteorológico Nacional).

En el verano, en evento El Niño la intensidad de la sequía interestival (canícula) se incrementa fuertemente mientras que en eventos de La Niña, la cantidad de precipitación se presenta 
arriba de lo normal (Pereyra et al., 1994). Durante la época invernal, El Niño induce aumento de lluvias y descenso de la temperatura (Cavazos, 1997; Conde et al., 2006; Vázquez-Aguirre, 2000; Ponnette-González et al., 2010); mientras que durante eventos de La Niña se caracteriza por la ausencia de lluvias durante la misma época (Magaña et al., 2003) haciendo más marcada la sequía preestival.

En el periodo de transición entre estas dos estaciones se presentan granizadas y ondas de calor que ocurren entre los meses de marzo y junio y entre los meses de marzo a mayo, respectivamente (SPC-Ver, 2017). Estos fenómenos influyen en el desarrollo del café y los daños a las plantas dependen de la etapa fenológica en la que se encuentren.

\section{Generalidades del cultivo de café}

El café es un cultivo perenne por lo que su desarrollo vegetativo y reproductivo continúa durante toda la vida de la planta. Durante el año los cafetos pasan por varios estados fenológicos que dependen, entre otros factores, de las condiciones ambientales de cada región productora. Para el caso de Veracruz, este cultivo está expuesto a diversos fenómenos meteorológicos y climáticos.

En la fenología, los estados más comunes en el periodo productivo del café son las etapas de floración (marzo a abril), crecimiento y llenado del fruto (mayo a octubre) y maduración y cosecha (noviembre a febrero) (Villers et al., 2009). El café requiere de una precipitación anual óptima de entre 1400-2300 mm y que la temperatura media oscile entre 17-23 ${ }^{\circ} \mathrm{C}$. De acuerdo con su ciclo fenológico, este cultivo necesita de un periodo de estrés por un déficit hídrico, de por lo menos 3 meses seguido de lluvias, para romper la latencia e iniciar su periodo productivo (Lin et al., 2008). Presenta una cosecha anual que inicia a finales de año y finaliza a inicios del siguiente año.

\section{Impactos de los fenómenos meteorológicos en las etapas fenológicas del café}

Son diversos los fenómenos meteorológicos que se presentan en la zona central de Veracruz que podrían afectar el cultivo de café y el nivel de afectación depende de la etapa de desarrollo en la que se encuentre el cultivo (Cuadro 1). Las granizadas, de acuerdo con el Calendario de Temporadas y Fenómenos Meteorológicos para el Estado de Veracruz de la Secretaría de Protección Civil, se presentan entre marzo y junio. Este fenómeno podría ocasionar no sólo 
daño mecánico en las plantas, sino tirar la flor y el grano, ya que durante este periodo el café se encuentra en floración y en las primeras etapas de desarrollo del fruto. El mismo efecto es producido por las lluvias torrenciales (Läderach et al., 2013; Ruelas-Monjardín, Nava-Tablada, Cervantes y Barradas, 2014).

Tabla 1.

Ocurrencia de Fenómenos Meteorológicos Durante las Diferentes Etapas Fenológicas del Café en Condiciones Agricológicas de la Zona Centro del Estado de Veracruz, México

Fenómenos Ene Feb Mar Abr May Jun Jul Ago Sep Oct Nov Dic

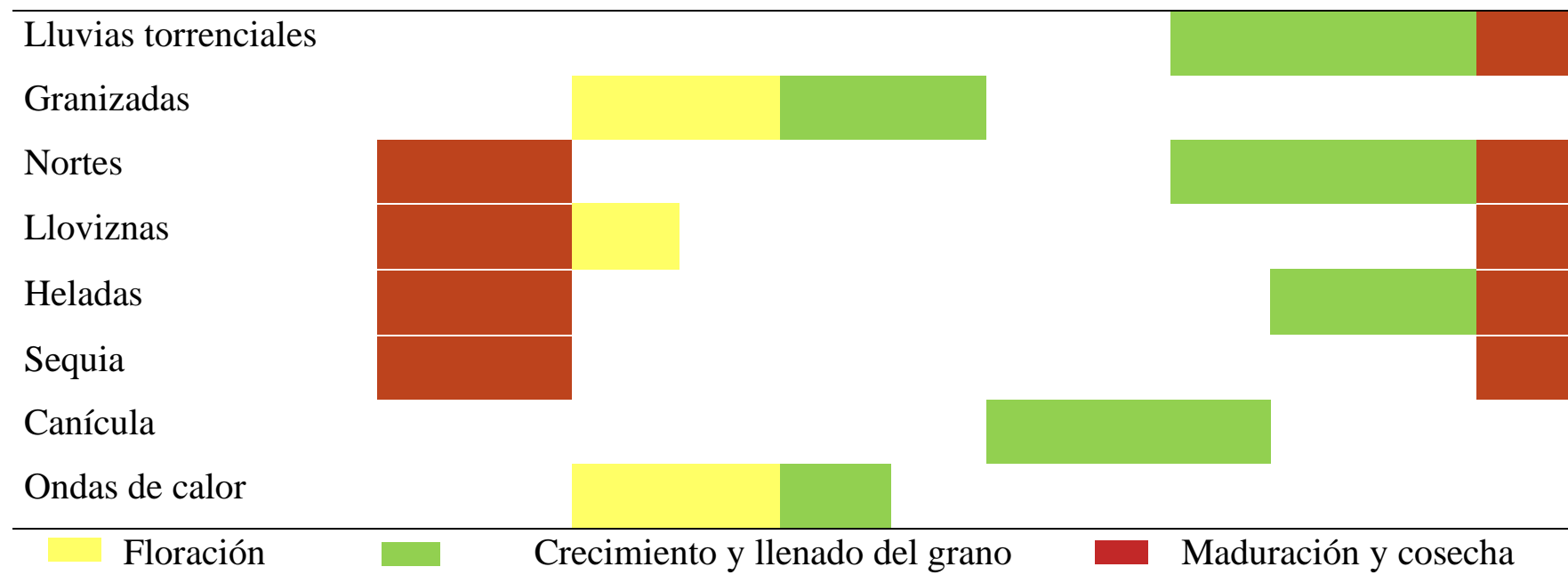

Fuente: Creación propia.

Los nortes, asociados a frentes fríos, son vientos intensos que dañan las plantas de café, de manera directa ya que ocasionan la caída de hojas y de los frutos maduros o en proceso de maduración, e indirectamente porque provocan la caída de ramas e incluso de árboles de sombra lo cual daña el cultivo. Los nortes generan descensos de temperatura y, si son muy marcados, los frutos y las hojas de las plantas se ven gravemente afectadas ya que por debajo de los $10{ }^{\circ} \mathrm{C}$ genera clorosis y quemaduras en el fruto (Damatta y Ramalho, 2006), afectando la producción debido a que se presenta cuando la cereza de café está en etapa de maduración.

De manera similar, las heladas ocasionan clorosis de las hojas y muerte de brotes (Pérez y Geissert, 2006); tienen un impacto directo en la producción del año en que se presentan al reducir su valor comercial ya que el tejido del fruto muere y el grano se oscurece. Si son muy severas, pueden afectar la producción de los años subsecuentes al obligar al rejuvenecimiento o renovación de la planta (Wintgens, 2004). Estos eventos no son frecuentes ni tan intensos en la zona, sin embargo, en el pasado, específicamente en el invierno de 1989, han tenido gran 


\section{Observatorios universitarios - Observatorio de la Cafeticultura Veracruzana}

impacto en la producción de café no sólo en el estado sino a nivel nacional (Pérez, 1996), siendo un factor que influyó en la crisis del café en México presentada ese mismo año (Vásquez, 2014).

Las ondas de calor se presentan generalmente entre los meses de marzo a mayo. Su intensidad y duración podría ocasionar el marchitamiento de plantas y flores ya que el café se encuentra en la etapa de floración (febrero-marzo) (Cuadro 1); así mismo, podría afectar el llenado del grano, si se encuentra en una etapa incipiente, pudiendo ser un grano vano. A mayor escala, durante la canícula las condiciones térmicas y de humedad son favorables para la proliferación de plagas. La intensidad de esta sequía podría influir en el llenado sólido del grano de café, impactando en su calidad.

\section{El niño y La niña: su influencia en la producción de café}

Las condiciones climáticas pueden ser perturbadas por fenómenos como El Niño Oscilación del Sur (ENSO). Varios estudios han reportado que la variabilidad interanual en el clima está relacionada en gran medida con el fenómeno El Niño Oscilación del Sur (ENSO) en su fase cálida “El Niño” y fría "La Niña. En México, la variabilidad de la precipitación está en buena medida modulada por este fenómeno (Seager et al., 2009; Tejeda-Martínez et al., 2011).

Existe evidencia de que eventos pasados de ENSO han influido en variaciones significativas en la producción de café en el estado de Veracruz (Conde et al., 2013). El aumento de lluvias y descenso de la temperatura durante la época invernal debido a este fenómeno son de gran importancia agrícola para esta zona, debido a que Veracruz es la segunda región más productora de café en México (SIAP). Las condiciones de sequía que se presentan entre los meses de diciembre y abril ocasionan estrés hídrico en las plantas que permite estimular la floración y el brote de yemas en las plantas de café (Alvim, 1960, citado en Haggar y Schepp, 2012; Villers et al., 2009).

Por el contrario, el exceso de agua derivado del incremento de la precipitación afecta negativamente el desarrollo del café ya que se presentan anomalías de floraciones anticipadas y dispersas, de forma que se incrementan los periodos de maduración y cosecha. Y si se extienden hasta inicios de primavera, el exceso de humedad podría favorecer la propagación de plagas y enfermedades, impactando en la producción. En contraparte, durante eventos de La Niña se caracteriza por una sequía más marcada (Magaña et al., 2003) y que de presentarse 


\section{Observatorios universitarios - Observatorio de la Cafeticultura Veracruzana}

posteriormente el inicio de las lluvias, favorecería a la mayor presencia de floración, lo que estaría relacionado con una mayor producción (Figura 2).

En primavera, el café requiere de humedad para la apertura floral y el cuajamiento del fruto. Un déficit de agua y aumento de temperatura, producido por el evento El Niño, ocasionaría flores inconclusas y el secado de frutos tiernos. Esto ha afectado la producción de café en la región (Conde et al., 2013; Gay et al., 2006), particularmente visto el periodo productivo 2002 3003 y 2015-2016, años en que se presentaron pérdidas de entre 22\% y 30\% en la producción de café en el estado de Veracruz; en el último periodo, debidas además a un brote atípico de roya presentado desde el 2012 (FIRA, 2016).

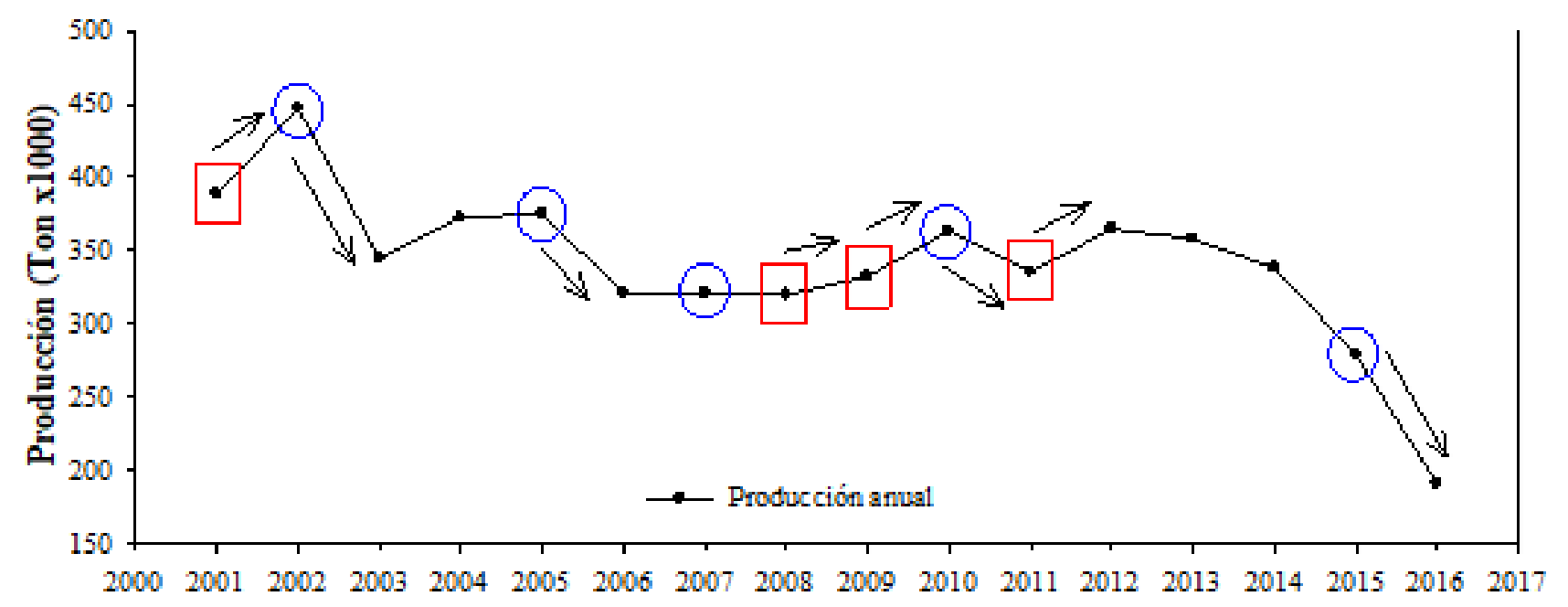

Figura 2. Producción anual de café en el estado de Veracruz (periodo 2001-2016). Periodos de Niño (Círculos) y Niña (Cuadros) al final del invierno y principios de la primavera y su efecto en el incremento o decremento de la producción anual en el estado de Veracruz (flechas). Fuente: Elaborado a partir de información del SIAP y la Administración Nacional Oceánica y Atmosférica (NOAA, por sus siglas en inglés).

En verano, la intensidad de la sequía interestival (canícula) se incrementa fuertemente (Pereyra et al., 1994). Esto impacta negativamente en la producción de café ya que se presentan durante el llenado del fruto, teniendo diferentes efectos por ejemplo que el grano no crezca, que se desarrolle vacío o que se forme sólo parcialmente. Por el contrario, en veranos con eventos de La Niña, el incremento de agua debida a la precipitación puede exceder el límite óptimo para el café pudiendo causar daños importantes, sumado a que las plantas podrían ser afectadas por tormentas severas y saturación de agua en el suelo. 
Ante ello, el mantenimiento y promoción de los árboles de sombra es una estrategia recomendada en la agricultura. Estos, aparte de funcionar como reservorios de carbono, permiten mitigar las inclemencias de los fenómenos meteorológicos atenuando los impactos de la intensidad de la precipitación y temperaturas extremas.

\section{Conclusión}

Los cultivos de café en la zona central de Veracruz son expuestos a una variedad y frecuencia de fenómenos meteorológicos que pueden afectar la producción y cosecha de las cerezas. Estos fenómenos pueden intensificarse o atenuarse con la presencia del ENSO, acrecentando los efectos en el cultivo. El café es más sensible cuando se encuentra en las etapas de floración y el inicio del crecimiento del fruto.

Sin embargo, no debe pasar inadvertido que la maduración, influenciada por temperaturas altas y condiciones de sequía, se vería severamente afectada, impactando en la producción y calidad del grano. No obstante, se deben realizar estudios más detallados a nivel de finca y a escala de paisaje de cómo pueden ser estas afectaciones en cada una de las etapas fenológicas del cultivo, ya que, en México, estos cultivos se desarrollan principalmente bajo árboles de sombra (sistemas agroforestales), esta condición podría ayudar a disminuir el impacto de la variabilidad climática producto de los fenómenos meteorológicos y por ende proteger a los cafetos durante las etapas más vulnerables.

\section{Referencias}

Báez, A. P., Padilla, H., Cervantes, J., Pereyra, D., \& Belmont, R. (1997). Rainwater chemistry at the eastern flanks of the Sierra Madre Oriental, Veracruz, Mexico. Journal of Geophysical Research: Atmospheres, 102(D19), 23329-23336. doi:10.1029/97JD02077

Barradas, V. L., Cervantes-Pérez, J., Ramos-Palacios, R., Puchet-Anyul, C., Vázquez-Rodríguez, P., \& Granados-Ramírez, R. (2010). Meso-scale climate change in the central mountain region of Veracruz State, México. En L. Bruijnzeel, F. Scatena, \& L. Hamilton (Eds.), Tropical Montane Cloud Forests (pp. 549-556). Cambridge University Press. doi:10.1017/CBO9780511778384.058

Bunn, C., Läderach, P., Rivera, O., \& Kirschke, D. (2015). A bitter cup: climate change profile of global production of Arabica and Robusta coffee. Climatic Change, 129(1-2), 89-101. doi:10.1007/s10584-014-1306-X 
Observatorios universitarios - Observatorio de la Cafeticultura Veracruzana

Cavazos, T. (1997). Downscaling large-scale circulation to local winter rainfall in north-eastern Mexico. International Journal of Climatology, 17(10), 1069-1082. doi:10.1002/(SICI)10970088(199708)17:10\%3C1069:AID-JOC183\%3E3.0.CO;2-I

Conde, C., Ferrer, R., \& Orozco, S. (2006). Climate change and climate variability impacts on rainfed agricultural activities and possible adaptation measures. A Mexican case study. Atmósfera, 19(3), 181-194. Recuperado de https://bit.ly/2VlYpZu.

Conde, C., Vinocur, M., Gay-García, C., Seiler, R., \& Estrada, F. (2013). Climatic threat spaces in Mexico and Argentina (pp. 279-306). En Climate Change and Vulnerability. CRC, Taylor and Francis. doi:10.4324/9781315067179.

DaMatta, F. M., \& Ramalho, C. J. D. (2006). Impacts of drought and temperature stress on coffee physiology and production: A review. Brazilian Journal of Plant Physiology, 18(1), 5581. doi:10.1590/S1677-04202006000100006

Diaz, H., \& Markgraf, V. (eds) (2000). El Niño and the Southern Oscillation: Multi-Scale Variability and Global and Regional Impacts. Journal of Quaternary Science, 18(5), 467496, doi:10.1002/jqs.775

Díaz-Padilla, G., Guajardo, R., \& López, R. (2013). Potencial productivo del cultivo del café en México (pp. 35-54). En López, R., Díaz, G., \& Zamarripa, A. (eds). El sistema producto café en México: problemática y tecnología de reproducción. CDMX, México: SAGARPAINIFAP.

Dilley, M., \& Heyman, B. (1995). ENSO and disaster: droughts, floods and El Niño/ Southern Oscillation warm events. Disasters, 19(3), 181-193, doi:10.1111/j.1467-7717.1995.tb00338.x

Fideicomisos Instituidos en Relación con la Agricultura (FIRA). 2016. Panorama Agroalimentario: Café 2016. Fideicomisos Instituidos en Relación con la Agricultura. Dirección de Investigación y Evaluación Económica y Sectorial. México, D.F., México. 37.

Gay, C., Estrada, F., Conde, C., Eakin, H., \& Villers, L. (2006). Potential impacts of climate change on agriculture: A case of study of coffee production in Veracruz, Mexico. Climatic Change, 79(3), 259-288. doi:10.1007/s10584-006-9066

Haggar, J., \& Schepp, K. (2012). Coffee and Climate Change. Impacts and options for adaption in Brazil, Guatemala, Tanzania and Vietnam. Climate Change, Agriculture and Natural Resource. Recuperado de https://bit.ly/340ohA5

Holwerda, F., Bruijnzeel, L. A., Muñoz-Villers, L. E., Equihua, M., \& Asbjornsen, H. (2010). Rainfall and cloud water interception in mature and secondary lower montane cloud forests of central Veracruz, Mexico. Journal of Hydrology, 384(1-2), 84-96. doi:10.1016/j.jhydrol.2010.01.012 
IPCC [Intergovernmental Panel on Climate Change]. (2007). Cambio climático 2007. Informe de síntesis. Contribución de los Grupos de trabajo I, II y III al Cuarto Informe de Evaluación del Grupo Intergubernamental de Expertos sobre el Cambio Climático. Ginebra, Suiza: IPCC. doi:10.1038/446727a

Jaramillo, J., Chabi-olaye, A., Kamonjo, C., Jaramillo, A., Vega, F., Poehling, H., \& Borgemeister, C. (2009). Thermal tolerance of the coffee berry borer Hypothenemus hampei: predictions of climate change impact on a tropical insect pest. Plos One, 4(8), e6487. doi:10.1371/journal.pone.0006487

Läderach, P., Haggar, J., Lau, C., Eitzinger, A., Ovalle, O., Baca, M., \& Lundy, M. (2013). Mesoamerican coffee: Building a climate change adaptation strategy. CIAT Policy Brief (2). Cali, Colombia: International Center for Tropical Agriculture (CIAT). Recuperado de https://hdl.handle.net/10568/29001

Lin, B. B., Perfecto, I., and Vandermeer, J. (2008). Synergies between agricultural intensification and climate change could create surprising vulnerabilities for crops. Bioscience. 58(9): 847-854. DOI: 10.1641/B580911.

Magaña, R. V., Vázquez, J. L., Pérez, J. L., \& Pérez, J. B. (2003). Impact of El Niño on precipitation in México. Geofísica Internacional, 42(3), 313-330. Recuperado de https://bit.ly/3apWF5n.

Magaña, R. V. (2004). Los impactos del niño en México. Centro de Ciencias de la Atmósfera, Universidad Nacional Autónoma de México, Secretaría de Gobernación. México.

Muñoz-Villers, L. E., Holwerda, F., Gómez-Cárdenas, M., Equihua, M., Asbjornsen, H., Bruijnzeel, L.A., Marín-Castro, B. E., \& Tobón, C. (2012). Water balances of old-growth and regenerating montane cloud forests in central Veracruz, Mexico. Journal of Hydrology, 462-463, 53-66. doi:10.1016/j.jhydrol.2011.01.062

Pereyra, D. D., Córdova, A. C. \& Palma, B. (1994). Effect of ENSO on the mid-summer drought in Veracruz, State, México. Atmósfera, 7(4), 211-219. Recuperado de file:///C:/Users/UV/Downloads/8376-8246-0-PB\%20(3).pdf

Pérez, G. I. (1996). Major cold air outbreaks affecting coffee and citrus plantations in the eastern and northeastern Mexico. Atmósfera, 9(1), 47-68. Recuperado de file:///C:/Users/UV/Downloads/8397-8267-0-PB.pdf

Pérez, P. E. \& Geissert, K. D. (2006). Zonificación agroecológica de sistemas agroforestales: el caso café (Coffea arabica L.) - Palma Camedor (Chamaedorea elegans Mart.). Inerciencia, 31(8), 556-562. Recuperado de https://www.redalyc.org/pdf/339/33911902.pdf

Ponnette-González, A. G., Weathers, K. C., \& Curran, L. M. (2010). Water inputs across a tropical montane landscape in Veracruz, Mexico: synergistic effects of land cover, rain and fog seasonality, and interannual precipitation variability. Global Change Biology, 16(3), 946963. doi:10.1111/j.1365-2486.2009.01985.x 
Observatorios universitarios - Observatorio de la Cafeticultura Veracruzana

Ruelas-Monjardín, L. C., Nava-Tablada, M. E., Cervantes, J., \& Barradas, V. L. (2014). Importancia ambiental de los agroecosistemas cafetaleros bajo sombra en la zona central montañosa del estado de Veracruz, México. Madera y Bosques, 20(3), 27-40. doi:10.21829/myb.2014.203149

Seager, R., Ting, M., Davis, M., Cane, M., Naik, N., Nakamura, J., Li, C., Cook, E., \& Stahle, D. W. (2009). Mexican drought: an observational modeling and tree ring study of variability and climate change. Atmósfera, 22(1), 1-13. Recuperado de https://bit.ly/2VESqOd.

Secretaria de Protección Civil, Veracruz. 2017. Calendario de Temporadas y Fenómenos Meteorológicos para el Estado de Veracruz de la Secretaría de Protección Civil. Recuperado de https://bit.ly/2KeAJ2L.

Tejeda-Martínez, A., Torres-Alavez, J. A., Ruiz-Barradas, A., Miranda, A. S. \& Salazar L. S. (2011). Evaluations and perceptions of the climate change in the State of Veracruz: an overview. En J. Blanco y H. Kherandmand (editores), Climate change: socioeconomic effects. (pp.131-155). Croacia: INTECH. ISBN 9789533074115.

Torres-Alavez, J. A., Tejeda, M. A., Vázquez, A. J., Brunet, I. M., Hernández, Á. P. \& Ruiz, B. A. (2010). Índices de cambio climático y análisis de variabilidad en el estado de Veracruz, México. En F. Fernández-García, E. Galán-Gallego y R. Cañada-Torrecilla (Eds). Ciudad, clima y ecosistemas (pp. 295-304). España: Asociación Española de Climatología

Vázquez-Aguirre, J. L. (2000). Caracterización objetiva de los nortes del Golfo de México y su variabilidad interanual (Tesis de Licenciatura). Facultad de Instrumentación Electrónica y Ciencias Atmosféricas de la Universidad Veracruzana. Xalapa, Ver.

Vásquez, P. J. (2014). Crisis del café y cultura del trabajo en el contexto de la acumulación flexible en el centro de Veracruz, México. Entorno Geográfico, 10, 136-151. Recuperado de https://bit.ly/3bzG5Bx

Villers, L., Arizpe, N., Orellana, R., Conde, C., \& Hernández, J. (2009). Impactos del cambio climático en la floración y desarrollo del fruto del café en Veracruz, México. Interciencia, 34(5), 322-329. Recuperado de http://ve.scielo.org/scielo.php?pid=S037818442009000500006\&script=sci_abstract

Wintgens, J. N. 2004. Factor influencing the quality of green coffee. En: J. N. Wintgens (ed.). Coffee: growing, processing, sustainable production, (789-809). Alemania: Weinhein, Wiley-VCH.

Zarazúa, V. P., Ruiz, C. J., Ramírez, O. G., Medina, G. G., Rodríguez, M. V., de la Mora, O. C., ... Durán, P. N. (2014). Índices de extremos térmicos en las Llanuras Costeras del Golfo Sur en México. Revista Mexicana de Ciencias Agrícolas, (10), 1843-1857. Recuperado de https://bit.ly/2VnDPYG. 